\title{
Intermittent fasting and adding more days to life
}

\author{
Abdul Kader Mohiuddin
}

\begin{abstract}
Department of Pharmacy, World University of Bangladesh, 151/8, Green Road, Dhanmondi, Dhaka - 1205, Bangladesh. Email: mohiuddin3@pharmacy.wub.edu.bd, Tel: +8801716477485.

Copyright @ 2019 Mohiuddin. This article remains permanently open access under the terms of the Creative Commons Attribution License 4.0, which permits unrestricted use, distribution, and reproduction in any medium, provided the original work is properly cited.
\end{abstract}

Received 22nd July, 2019; Accepted 27th July, 2019

\section{Respected sir,}

With over $50 \%$ of the adult population in the UK currently classified as overweight or obese, accompanied by an increased risk of type 2 diabetes and cardiovascular disease, finding more effective strategies to manage these conditions remains imperative (Ganesan et al., 2018). In 2016, World Health Organization (WHO) reported that more than 1.9 billion people in the world were overweight and over 650 million people were obese which has tripled in number since 1975 (Harvie and Howell, 2017). To date, the most robust intervention efficient in warding off the aforementioned cellular markers of aging is calorie restriction (CR) that involves the administration of a wellbalanced, nutrient-dense diet that reduces calorie intake by 20 to $40 \%$ without malnutrition (Picca et al., 2017). It is the only non-genetic intervention that has consistently been found to extend both mean and maximal life span across a variety of species (Anton and Leeuwenburgh, 2013). Randomized trials demonstrate that intentional weight loss reduces type 2 diabetes, retards aging-related functional decline; and increases cognitive and physical function; and increases lifespan (Templeman et al., 2018). Modest weight losses of 5 to $10 \%$ have been associated with significant improvements in cardiovascular disease risk factors (ie, decreased $\mathrm{HbA} 1 \mathrm{C}$ levels, reduced blood pressure, increase in HDL cholesterol, decreased plasma triglycerides) in patients with T2D (Furmli et al., 2018). In addition to the weight loss effects and metabolic improvements, several other beneficial effects of therapeutic fasting have been described including improvements in lipid profiles, osteoarthritis, healing of thrombophlebitis, healing of refractory dermal ulcers and tolerance of elective surgery (Anton et al., 2018). Recent findings indicate that meal timing is crucial, with both intermittent fasting and adjusted diurnal rhythm of feeding improving health and function, in the absence of changes in overall intake. Lowered intake of particular nutrients, rather than of overall calories, is also key, with protein and specific amino acids playing prominent roles (Fontana and Partridge, 2015). Importantly, long-term exposure to a CR diet might also cause substantial side effects like amenorrhea, osteoporosis, decreased fertility and libido (due to reduced testosterone in men), impaired wound healing and increased susceptibility to infections (Rusli $F$ et al., 2017; Malinowski et al., 2019). Fasting is distinct from caloric restriction (CR), in which daily caloric intake is chronically reduced by up to $40 \%$, but meal frequency is maintained (Longo and Mattson, 2014). The cellular and molecular mechanisms by which Intermittent Fasting (IF) improves health and counteracts disease processes involve activation of adaptive cellular stress response signaling pathways that enhance mitochondrial health, DNA repair and autophagy (Mattson et al., 2017). IF regimens that induce the metabolic switch have the potential to improve body composition in overweight individuals. Moreover, IF regimens also induce the coordinated activation of signaling pathways that optimize physiological function, enhance performance, and slow aging and disease processes (Anton et al., 2018). Current evidence suggests that as little as $10 \mathrm{~min}$ of high intensity exercise can improve metabolic health and aerobic capacity and alternative day fasting can reduce obesityassociated changes in body composition, fasting insulin and glucose concentrations (Mattson et al., 2017). Studies in rodents have demonstrated that restricting the availability of food to the normal nighttime feeding cycle improves metabolic profiles and reduces the risk of obesity and obesity-related conditions, such as nonalcoholic fatty liver disease, and chronic diseases, such as diabetes and cancer (Wilson et al., 2018). Fasting periods with various patterns are found in most religions. In fact, this ascetic practice is referenced in the Old Testament, as well as other ancient texts such the Koran and the Mahabharata. Muslims, for example, fast from dawn until dusk during the month of Ramadan, while Christians, Jews, Buddhists, 
and Hindus traditionally fast on designated days or periods. For instance, Ramadan intermittent fasting was linked with improvements in cardiometabolic risk factors (Patterson and Sears, 2017; Moro et al., 2016). Studies reported that total cholesterol (TC), low-density lipoprotein (LDL), high-density lipoprotein (HDL) and blood glucose have been improved after Ramadan compared to before Ramadan among athletes. Rahbar et al. (2019) concluded that fasting in Ramadan independent of anthropometric measures decreases IGF-1, IL-2, and serum lipid levels (de Toledo et al., 2019). As Smocking has been forbidden during fasting of Ramadan, studies revealed a significant reduction in second-hand smoke levels in public places (Rahbar et al., 2019). Even with no advice on lifestyle changes, there are consistent-albeit transient-reductions in weight and fat mass with the Ramadan fast, especially in people with overweight or obesity (Rouhani and Azadbakht, 2014). Ramadan intermittent fasting might be associated with decrease in sexual desire, frequency of sexual intercourse and serum FSH level (Talib et al., 2015). Remarkably, IF during early adulthood, and also during mid-life, was sufficient to extend lifespan, indicating a "memory" effect hormesis (a phenomenon by which "lowlevel" toxic stress elicits response mechanisms that protect against similar but higher-level stresses associated with aging). Given that intermittent starvation in early life led to increased post-IF starvation resistance in addition to increased lifespan, hormesis could play a role (Fernando et al., 2019). One of metabolic effects of intermittent fasting is intermittent ketosis known for its appetite suppression effect resulting in voluntary calorie reduction. In terms of meal timing, skipping breakfast is similar to intermittent fasting (Catterson et al., 2018). Although, several studies reported associations between breakfast skipping and fatigue at noon, worsens memory and higher body mass index as well as increased prevalence of obesity-related chronic illness; deficient in total energy, vitamins and minerals, increased risk of central adiposity, and risk of insulin resistance and cardio-metabolic disorders (Mohiuddin, 2019). There are two basic varieties of the IF diet. The most popular variation is time-restricted feeding. It may be used in three variants: $16 / 8,18 / 6$ and 20/4. 16:8, consisting of a 16-h fast, and then an 8-h nutritional window. Another protocol consists of a 24-h fasting period, alternated with a 24-h eating period, repeated two or three times a week. There are two possible systems, 5:2 or 4:3. In the 5:2 system, in which caloric restriction is used for two days a week, and a regular diet for 5 days (Johnstone, 2015; Harvie and Howell, 2017). However, Intermittent fasting should not be used by children, pregnant women, and people performing heavy physical work (Ganesan et al., 2018). Intermittent fasting would be a useful tool in distressful condition to improve learning and memory by downregulation of the putative molecular factors involved in neuro-inflammation, although chronic stressors are generally well-known for adverse effects on the body particularly cognitive decline (Shojaie et al., 2017). To improve health, the goal should be to lose weight by reducing the total amount of calories consumed, rather than focusing on when those calories are consumed (Shojaie et al., 2017). Four to eight hours time restricted feeding reduces caloric intake (without calorie counting) and significantly decreases fat mass without changing lean mass in young resistance trained men. Gabel et al. (2018) revealed that 8 hours time restricted feeding produces mild caloric restriction and weight loss, without calorie counting. It may also offer clinical benefits by reducing blood pressure (Gabel et al., 2018). Altering body composition in such a manner may be advantageous to the athlete for various biomechanical, aesthetic, and locomotive reasons, thereby increasing the likelihood of competitive success in a target weight-class (e.g., combat sports, weight lifting), weight-sensitive sports (e.g., endurance events, ski jumping), or aesthetically judged sports (e.g., gymnastics and bodybuilding) (Peos et al., 2019). IF regimens may be a promising approach to lose weight and improve metabolic health for people who can tolerate intervals of not eating, or eating very little, for certain hours of the day or days of the week. If proven to be efficacious, these eating regimens may offer promising nonpharmacologic approaches to improving health at the population level with multiple public health benefits (Patterson et al., 2015).

\section{CONFLICT OF INTEREST}

The author declares no competing interests.

\section{REFERENCES}

Anton, S. D., Moehl, K., Donahoo, W. T., Marosi, K., Lee, S. A., Mainous III, A. G., Leeuwenburgh, C., \& Mattson, M. P. (2018). Flipping the metabolic switch: understanding and applying the health benefits of fasting. Obesity, 26(2), 254-268.

Anton, S., \& Leeuwenburgh, C. (2013). Fasting or caloric restriction for healthy aging. Experimental Gerontology, 48(10), 1003-1005.

Catterson, J. H., Khericha, M., Dyson, M. C., Vincent, A. J., Callard, R., Haveron, S. M., Rajasingam, A., Ahmad, M., \& Partridge, L. (2018). Short-term, intermittent fasting induces long-lasting gut health and TOR-independent lifespan extension. Current Biology, 28(11), 1714-1724.

de Toledo, F. W., Grundler, F., Bergouignan, A., Drinda, S., \& Michalsen, A. (2019). Safety, health improvement and wellbeing during a 4 to 21-day fasting period in an observational study including 1422 subjects. PloS one, 14(1), e0209353.

Fernando, H. A., Zibellini, J., Harris, R. A., Seimon, R. V., \& Sainsbury, A. (2019). Effect of Ramadan Fasting on Weight and Body Composition in Healthy Non-Athlete Adults: A Systematic Review and Meta-Analysis. Nutrients, 11(2), 478.

Fontana, L., \& Partridge, L. (2015). Promoting health and longevity through diet: from model organisms to humans. Cell, 161(1), 106-118.

Furmli, S., Elmasry, R., Ramos, M., \& Fung, J. (2018). Therapeutic use of intermittent fasting for people with type 2 diabetes as an alternative to insulin. MBJ Case Reports, Pp. 14. 
Gabel, K., Hoddy, K. K., Haggerty, N., Song, J., Kroeger, C. M., Trepanowski, J. F., Panda, S., \& Varady, K. A. (2018). Effects of 8-hour time restricted feeding on body weight and metabolic disease risk factors in obese adults: A pilot study. Nutrition and Healthy Aging, 4(4), 345-353.

Ganesan, K., Habboush, Y., \& Sultan, S. (2018). Intermittent fasting: The choice for a healthier lifestyle. Cureus, 10(7), e2947.

Harvie, M., \& Howell, A. (2017). Potential benefits and harms of intermittent energy restriction and intermittent fasting amongst obese, overweight and normal weight subjects - a narrative review of human and animal evidence. Behavioral Sciences, 7(1), 22p.

Johnstone, A. (2015). Fasting for weight loss: an effective strategy or latest dieting trend? International Journal of Obesity, 39(5), 727-733.

Longo, V. D., \& Mattson, M. P. (2014). Fasting: molecular mechanisms and clinical applications. Cell Metabolism, 19(2), 181-192.

Malinowski, B., Zalewska, K., Węsierska, A., Sokołowska, M. M., Socha, M., Liczner, G., Pawlak-Osińska, K., \& Wiciński, M. (2019). Intermittent Fasting in Cardiovascular Disorders-An Overview. Nutrients, 11(3), 673.

Mattson, M. P., Longo, V. D., \& Harvie, M. (2017). Impact of intermittent fasting on health and disease processes. Ageing Research Reviews, 39, 46-58.

Mohiuddin, A. K. (2019). Skipping Breakfast Everyday Keeps Well-Being Away. Research \& Reviews: Journal of Dairy Science and Technology, 7(3), 20-30.

Moro, T., Tinsley, G., Bianco, A., Marcolin, G., Pacelli, Q. F., Battaglia, G., Palma, A., Gentil, P., Neri, M., \& Paoli, A. (2016). Effects of eight weeks of time-restricted feeding (16/8) on basal metabolism, maximal strength, body composition, inflammation, and cardiovascular risk factors in resistancetrained males. Journal of translational medicine, 14(1), 290.

Patterson, R. E., \& Sears, D. D. (2017). Metabolic effects of intermittent fasting. Annual Review of Nutrition, 37, 371-393.

Patterson, R. E., Laughlin, G. A., LaCroix, A. Z., Hartman, S. J., Natarajan, L., Senger, C. M., Martínez, M. E., Villaseñor, A., Sears, D. D., Marinac, C. R., \& Gallo, L. C. (2015). Intermittent fasting and human metabolic health. Journal of the Academy of Nutrition and Dietetics, 115(8), 1203-1212.
Peos, J. J., Norton, L. E., Helms, E. R., Galpin, A. J., \& Fournier, P. (2019). Intermittent Dieting: Theoretical Considerations for the Athlete. Sports, 7(22), 26p.

Picca, A., Pesce, V., \& Lezza, A. M. S. (2017). Does eating less make you live longer and better? An update on calorie restriction. Clinical Interventions in Aging, 12, 1887-1902.

Rahbar, A. R., Safavi, E., Rooholamini, M., Jaafari, F., Darvishi, S., \& Rahbar, A. (2019). Effects of intermittent fasting during ramadan on insulin-like growth factor-1, interleukin 2 , and lipid profile in healthy muslims. International journal of preventive medicine, 10, 7

Rouhani, M. H., \& Azadbakht, L. (2014). Is Ramadan fasting related to health outcomes? A review on the related evidence. Journal of research in medical sciences: the official journal of Isfahan University of Medical Sciences, 19(10), 987892.

Rusli, F., Lute, C., Boekschoten, M. V., van Dijk, M., van Norren, K., Menke, A. L., Müller, M., \& Steegenga, W. T. (2017). Intermittent calorie restriction largely counteracts the adverse health effects of a moderate-fat diet in aging C57BL/6J mice. Molecular nutrition \& food research, 61(5), 1600677.

Shojaie, M., Ghanbari, F., \& Shojaie, N. (2017). Intermittent fasting could ameliorate cognitive function against distress by regulation of inflammatory response pathway. Journal of Advanced Research, 8(6), 697-701.

Talib, R. A., Canguven, O., Al-Rumaihi, K., Al Ansari, A., \& Alani, M. (2015). The effect of fasting on erectile function and sexual desire on men in the month of Ramadan. Urology journal, 12(2), 2099-2102.

Templeman, I., Thompson, D., Gonzalez, J., Walhin, J. P., Reeves, S., Rogers, P. J., Brunstrom, J.M., Karagounis, L. G., Tsintzas, K., \& Betts, J. A. (2018). Intermittent fasting, energy balance and associated health outcomes in adults: study protocol for a randomised controlled trial. Trials, 19(1), 86.

Wilson, R., Deasy, W., Stathis, C., Hayes, A., \& Cooke, M. (2018). Intermittent Fasting with or without Exercise Prevents Weight Gain and Improves Lipids in Diet-Induced Obese Mice. Nutrients, 10(3), 346. 